\title{
PERSPECTIVAS PARA O USO DA PESQUISA OBSERVACIONAL EM BIBLIOTECA UNIVERSITÁRIA: UM ESTUDO NA COLEÇÃO DE PERIÓDICOS
}

\author{
PERSPECTIVES FOR THE USE OF OBSERVATIONAL RESEARCH IN UNIVERSITY \\ LIBRARY: A STUDY IN THE COLLECTION OF PERIODICALS
}

PERSPECTIVAS PARA EL USO DE LA INVESTIGACIÓN OBSERVACIONAL EN BIBLIOTECA UNIVERSITARIA: UN ESTUDIO EN LA COLECCIÓN DE PERIÓDICOS

${ }^{1}$ José Aparecido Augusto, ${ }^{1}$ Ariadne Chloe Mary Furnival, ${ }^{1}$ Eliane Colepicolo, ${ }^{1}$ Ana Carolina Simionato.

${ }^{1}$ Universidade Federal de São Carlos

\section{Correspondência}

1José Aparecido Augusto

Universidade Federal de São Carlos

São Carlos, SP.

Email: joseaugusto.ufscar@gmail.com

ORCID: http://orcid.org/0000-0001-7893-1129

Submetido em: $19-10-2016$

Aceito em: 02-03-2017

Publicado: 07-06-2017

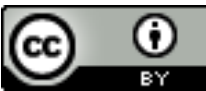

JITA: DD. Academic libraries. 
RESUMO: Analisa o uso da coleção de periódicos impressos numa biblioteca universitária, por meio de pesquisa observacional estruturada, sistemática e não participante e também do levantamento de dados estatísticos de uso, no período compreendido entre os anos de 2012 a 2015, junto à Biblioteca. Enquadra-se, academicamente, na grande área de "usos da informação e acervos", com inspiração na contribuição intelectual de Lancaster e de outros autores no campo de estudos de usuários. A partir dos dados obtidos, espera-se contribuir para um possível estabelecimento de uma política para readequação do espaço atualmente utilizado por este acervo de periódicos, visando colaborar com a comunidade acadêmica para estar em sintonia com as linhas de pesquisa e projetos desenvolvidos na Universidade.

PALAVRAS ChavE: Estudo de usuários. Usos da informação e acervos. Periódicos impressos: estudo de caso.

ABSTRACT: Reports on a study that analyzes the use of a collection of printed journals in a Brazilian university library, using structured and systematic non-participant observational research. The study is placed within the area of "information and collection use", inspired by the contributions of Lancaster, Wilson and other authors in the field of user and use studies. Use of this journal collection was also analyzed using the Library's own statistical data on collection use, for the period of 2012 to 2015. From the data obtained, we hope to contribute to the definition of policy parameters aiming to adjust the physical space currently used for this printed journal collection, aiming to collaborate so that the library might be more in tune with user needs and the research and projects developed in the University.

KEYWORDS: User studies. Uses of information and collections. Printed journals. Case study.

RESUMEN: Se analiza el uso de la colección de periódicos impresos en una biblioteca universitaria, por medio de una investigación observacional estructurada, sistemática y no participante y también del levantamiento de datos estadísticos de uso, en el período comprendido entre los años 2012 a 2015, junto a la Biblioteca. Se encuadra, académicamente, en la gran área de "usos de la información y acervos", con inspiración en la contribución intelectual de Lancaster y de otros autores en el campo de estudios de usuarios. A partir de los datos obtenidos, se espera contribuir a un posible establecimiento de una política para readecuación del espacio actualmente utilizado por este acervo de periódicos, con el fin de colaborar con la comunidad académica para estar en sintonía con las líneas de investigación y proyectos desarrollados en la Universidad.

PAlABRAS CLAVE: Estudio del usuario. Usos de la información y las colecciones. Revistas impresas: un estudio de caso. 


\section{INTRODUÇÃ̃o}

Os estudos de usuários cresceram muito nas últimas décadas, passando de uma abordagem voltada aos sistemas para um enfoque alternativo, no qual o usuário da informação torna-se o agente principal e sujeito ativo dos processos que envolvem a biblioteca. $\mathrm{O}$ uso de uma Unidade Informacional passou a considerar as necessidades informacionais do usuário e seu comportamento na busca pela informação, onde os dados qualitativos referentes a essas necessidades prevalecem sobre os dados quantitativos.

Hoje, o foco dos estudos de usos e usuários de bibliotecas e serviços de informação tem se voltado para a "nova" realidade: de que a maioria das pessoas não sente a necessidade de utilizar as bibliotecas - física e virtualmente - para conseguir a informação que precisa (CONNOWAY, 2015). Fala-se, na literatura, da "geração Google" e dos "screenagers" (adolescentes e jovens cujas vidas são permeadas por uma afinidade constante com a comunicação eletrônica por meio das telas do seu telefone, computador, televisão etc.), para quem o primeiro ponto de partida na busca pela informação sempre será a Internet, por meio de seus específicos motores de busca (CONNOWAY, 2015; DEMPSEY, 2015). As implicações disso, observa Connoway (2015), é que a biblioteca precisa se tornar enraizada nos hábitos e estilo de vida dos usuários e potenciais futuros novos usuários. De acordo com Dempsey (2015), este fato se traduzirá, na prática, pela necessidade de uma virada profunda de foco da biblioteca, que agora deverá estar "na vida do usuário" e não como antes, esperando que o usuário estivesse ou se encaixasse na vida da biblioteca.

Neste trabalho propomos analisar o usuário por meio de pesquisa observacional estruturada, sistemática e não participante (RICHARDSON, 1999; CUNHA, 1982), levantando o número de consulentes e tipo de material consultado e, com o auxílio de dados estatísticos, investigar e analisar o quanto foram utilizados os periódicos impressos disponibilizados numa biblioteca universitária. De fato, o próprio Ranganathan já tinha, em 1931, percebido que a observação do usuário constitui uma forma natural e potencialmente poderosa para entender o comportamento do usuário da biblioteca, com um olhar em como melhorar os serviços e produtos prestados pela mesma. Em relação à quarta lei ("Poupe o tempo do leitor"), Ranganathan observou que:

Talvez esta lei não seja tão evidente quanto as outras. No entanto, tem sido responsável por muitas reformas na administração de bibliotecas e tem um grande potencial para afetar muito mais reformas no futuro. Talvez o método mais conveniente de estudar as consequências desta lei será seguir um leitor desde o momento de sua entrada na biblioteca, até o momento em que ele a deixa, examinando criticamente cada processo, o que ele tem percorrido ou feito, com um olho para a economia de tempo que pode ser efetuada em cada fase. (RANGANATHAN, 1931, p. 337, grifo e tradução nossos).

Mesmo considerando-se toda a evolução tecnológica e os avanços nos estudos de comportamentos do usuário e da busca de informações, a moderna Biblioteconomia dificilmente poderá deixar de alicerçar-se nos ensinamentos deste grande pensador da área.

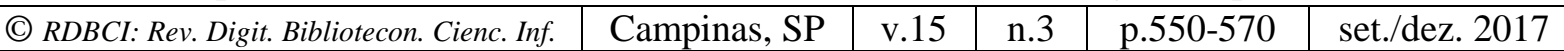


Mais recentemente, na literatura sobre a "experiência do usuário" - UX: User Experience - os métodos etnográficos de observação estão assumindo um papel central para desvelar os detalhes mais finos e as experiências do dia-a-dia dos usuários em relação aos serviços prestados pela biblioteca (PRIESTNER; BORG, 2016). Esta abordagem mais recente vem acrescentar e aprofundar a outra virada paradigmática do campo de estudos de usuários, que ocorreu nas décadas de 70 e 80, centrando-se no usuário ao invés do sistema ou na biblioteca e, na literatura, é usualmente referido como a "abordagem alternativa" ou “abordagem da percepção do usuário" (FERREIRA, 1997; GONÇALVES, 2013).

A pesquisa enquadra-se na grande área de "usos da informação e acervos", com inspiração em Lancaster (1993) e também busca referências na contribuição intelectual de autores no campo de estudo das necessidades e comportamento informacional, como Dervin e Taylor, entre outros. O objetivo é compreender e explicar aspectos relacionados às necessidades de informação dos usuários daquela biblioteca.

Tratou-se de uma pesquisa observacional estruturada, com preenchimento de um formulário previamente preparado para a pesquisa, cujos autores também foram responsáveis pelas observações empíricas deste estudo. Não se interferiu no meio ambiente observado, caracterizando-a, portanto, como uma pesquisa sistemática (NOGUEIRA, 1969, P. 82) e sem nenhuma tentativa de manipular variáveis, pois a pesquisa foi do tipo não participante.

Como unidade-caso, foi escolhida a Biblioteca Comunitária (BCo) da Universidade Federal de São Carlos (UFSCar). Inaugurada em 1995, a BCo apresentou-se como um projeto pioneiro no país, com a finalidade de democratizar seu espaço físico e acervo, seus produtos e serviços, visando atender à comunidade universitária e científica, bem como à comunidade externa em geral. Dessa forma, a BCo é também considerada um canal dinâmico e eficiente, catalisador da informação gerada e armazenada na Universidade (PADILHA; LIMA, 2006).

Em estudo publicado por Ninin et al (2015), no qual também foi selecionada como unidade-caso a BCo, os autores constataram que esta biblioteca contava com acervo de cerca de 241 mil exemplares de obras monográficas, além de mais de 4 mil títulos de periódicos e igual número de teses e dissertações. Além dessas categorias, o acervo compreende as coleções especiais, como o Fundo Florestan Fernandes e o acervo multimídia, dentre outros.

O presente estudo analisou particularmente o uso e usuários do acervo físico dos periódicos impressos, disponível para consulta numa biblioteca universitária e o problema apresentado: quanto foram usadas estas revistas impressas? O espaço ocupado por este acervo, atualmente, poderia ser readequado para outra finalidade? Os objetivos deste estudo foram analisar o uso e usuários do acervo físico dos periódicos impressos e disponíveis para consulta na BCo/UFSCar e apontar possibilidades de remanejamento na disposição física dos periódicos impressos e a utilização do espaço atualmente reservado a estes periódicos para outras necessidades da Biblioteca. 
Assim, buscou-se revisar os aspectos teóricos relativos à área de estudos dos usuários e, por meio da observação, compreender o uso que se faz do acervo em geral, o que determina o papel do bibliotecário nas atividades de suporte às atividades de ensino. Segundo Almeida (2000) o estudo é igualmente importante para que se observem quais são os objetivos da instituição e a opinião dos usuários que utilizam a biblioteca, de forma que sejam avaliados qualitativamente os produtos ou serviços de informação.

\section{ESTUDOS DE USOS E USUÁRIOS}

As pesquisas acerca do tema 'estudo de usuários', aparentemente, permanecem imutáveis há mais de 40 anos, com foco em coleta de dados para criar ou avaliar produtos e serviços informacionais, além de procurar entender melhor o fluxo da transferência da informação (BAPTISTA; CUNHA, 2007). Para Baptista e Cunha (2007) inúmeros trabalhos realizados nessas décadas resultaram em estudos que não podem ser comparados, principalmente devido à utilização de variadas técnicas de coleta de dados. Durante a década de 1940, surgiu outra grande contribuição para o estudo de usuários, que foi a mudança de perfil da biblioteca, de passiva, que pouco compreendia sobre o que o usuário sabia ou não fazer uso da informação disponível para ativa, a qual se preocupa com a melhoria dos serviços existentes e a criação de novos serviços como disseminação seletiva da informação, serviços de alerta, boletins, entre outros. (RIBEIRO; COSTA, 2011).

Figueiredo (1994) argumenta que os estudos de usos e usuários são canais de comunicação que se abrem entre a biblioteca e a comunidade à qual ela serve. E uma maneira mais conveniente de sua caracterização é dividi-los em dois tipos: 1) estudos orientados ao uso, com objetivos como a coleta de dados a fim de avaliar, melhorar ou criar serviços de informação; 2) estudos orientados ao usuário, cujos objetivos, entre outros, inclui procurar compreender melhor o fluxo da informação. Estes dois tipos de objetivos, numa pesquisa mais profunda, não são excludentes, pelo contrário, são complementares para que se obtenha uma visão mais ampla do serviço em estudo. "[...] há necessidade de mais estudos longitudinais para que se possam medir as variações de uso e necessidade de informação ao longo de um prazo maior." (BAPTISTA; CUNHA, 2007, p. 169). Desta maneira, torna-se importante sua diferenciação. Ninin et al (2015) lembram que os estudos de uso são elaborados para avaliar o quanto e como uma determinada coleção está sendo utilizada.

Lancaster (1993) considera que a avaliação de um serviço de informação pode ser subjetiva ou objetiva, enquadrando-se na grande área de 'usos da informação e acervos'. Os estudos subjetivos, baseados em opiniões, têm sua importância na medida em que se descobre os sentimentos dos usuários em relação ao serviço. Entretanto, se a avaliação for analítica e diagnóstica, procurando descobrir como o serviço poderia melhorar, sua utilidade passa a ser de máxima importância, pois para isso há necessidade de se adotar critérios e procedimentos objetivos, com resultados que podem ser quantificáveis. Lancaster (1993) constata a evidente diferença entre as medidas de uso na própria biblioteca e a medida de circulação registrada, 
demonstrando a ambiguidade da primeira: se um documento é ou não emprestado isto se constitui como uso? Se é retirado da estante, lido parcialmente e depois devolvido a seu lugar, terá sido usado? Não há como se ter certeza de que algum item seja utilizado dentro de uma biblioteca, sem que se entrevistem usuários representativos ou, pelo menos, sem que sejam observados por algum período de tempo razoável.

Figueiredo (1983) esclarece que as investigações sobre os estudos de usuários, os quais possuem como objetivo conhecer o que os indivíduos precisam em matéria de informação ou se suas necessidades de informação serão satisfeitas de maneira adequada, são os canais de comunicação que se abrem entre a biblioteca e a comunidade à qual ela serve. Além disso, estes estudos são, de fato, um método científico, pois cumprem com todos os requisitos postulados pelo pensamento científico, mas possuem a variável 'ser humano', o que os tornam passíveis de serem tratados como um fenômeno de ordem social. Portanto, "[...] é considerado científico como método e social como aplicação." (FIGUEIREDO, 1983, p. 43).

Objetivando o levantamento das preferências dos leitores, os primeiros estudos de usuário de bibliotecas aos poucos foram incorporando os métodos da ciência do comportamento, com preocupação em primeiro plano com a "[...] explanação dos fenômenos, a predição do uso e o controle da utilização da informação, através da manipulação das condições essenciais." (LIMA, 1974, p. 51). Análises de Pithan (2009) e também de Baptista e Cunha (2007), demonstram que no período compreendido entre as décadas de 60 e 80, as pesquisas tiveram como foco principal os estudos quantitativos, com intensa utilização das técnicas estatísticas, quer na coleta dos dados, quer em seu tratamento e teve "[...] por objetivo garantir uma maior precisão na análise e interpretação dos resultados, tentando, assim, aumentar a margem de confiabilidade quanto às inferências dos resultados encontrados. " (BAPTISTA; CUNHA, 2007, p. 170). Gradualmente, o usuário passa a apresentar-se como um sujeito participativo em praticamente todos os processos que envolvem a biblioteca. Isto fica ainda mais evidente no contexto de acervos digitais on-line, no qual o usuário passa a ser o próprio autor e gestor dos recursos digitais disponibilizados pela biblioteca (AOYAMA, 2011).

A literatura sobre necessidades de informação, por meio dos estudos de usuários, já acumulou grande número de pesquisas. Miranda (2006) lembra que essas pesquisas contam com revisões de literatura e análises publicadas no Annual Review of Information Science and Technology (ARIST) que são ricas em apontar características, tendências e importantes variáveis nos estudos de uso e necessidades de informação.

Estudos de Bettiol (1990) e Case (2012) afirmam que o conceito de necessidade de informação é amplo, difícil de definir, isolar ou medir, pois vários pesquisadores têm tentado interpretar o significado do termo "necessidade de informação" e, por ser um dos assuntos mais complexos da área de estudos de usuários, ainda não há uma definição satisfatória sobre este tema, o que nos levará a abordar as opiniões de vários autores. Para Figueiredo (1983), o 
conceito de necessidade de informação também se apresenta como um dos mais complexos no campo de estudo de usuários e, procurando trazer melhor compreensão ao tema, a autora baseia-se na definição de Line (1974), a qual argumenta que a necessidade é habitualmente arquitetada como uma contribuição para uma finalidade pertinente, não frívola, mesmo aquela com fim recreacional. Em relação à busca da informação, Choo (2000) observa que esta é "[...] o processo humano e social por meio do qual a informação se torna útil para um indivíduo ou grupo." (CHOO, 2000, p. 99) e propõe que, no processo de busca da informação, há divisão conceitual em três estágios: a clarificação da necessidade de informação, a busca e o uso da informação.

Choo (2000), utilizando a abordagem cognitiva de criação de significado desenvolvida e aplicada por Brenda Dervin, as reações emocionais que acompanham o processo de busca da informação relatadas por Carol Kuhlthau e a abordagem proposta por Robert Taylor sobre as dimensões situacionais do ambiente em que a informação é usada, Choo (2000) conclui que cada uma destas três perspectivas lança sua própria luz sobre as escolhas e ações nos principais estágios do comportamento no processo de busca da informação: clareza da necessidade, busca e uso da informação. Este comportamento ativo do indivíduo foi classificado por Ellis e outros autores como "[...] atividades de busca da informação em iniciar, encadear, vasculhar, diferenciar, monitorar, extrair, checar e terminar." (CHOO, 2000, p. 113, 114).

\section{PESQUISA OBSERVACIONAL NOS ESTUDOS DE USOS E USUÁRIOS}

Há um consenso na literatura de que os métodos e técnicas quantitativos têm predominado na área de estudos de usos e usuários. O uso e análise de estatísticas rotineiramente levantados pela biblioteca (indicadores do uso de certos itens no acervo, a frequência do uso de certas partes do acervo, a quantidade de visualizações do website da biblioteca, a quantidade de downloads de e-books, o número de usuários que passam pela entrada da biblioteca para entrar no prédio - em inglês, o indicador do footfall) é frequentemente conjugado com a coleta de dados diretos, junto aos usuários, por meio de questionários e entrevistas. Gonçalves (2013) sintetiza bem a cronologia desta tendência pela escolha desses métodos na área, que Chowdhury e Chowdhury (2011, p. 381) denominam como sendo "abordagens positivistas e reducionistas" e que Janes (2012) critica por não levarem a uma compreensão sofisticada do uso da informação. Isto se deve ao fato de que os questionários tendem a ser respondidos por usuários que já são mais "a favor" do uso da biblioteca, e que os autorrelatos sobre o próprio comportamento do usuário não são inteiramente confiáveis. Em relação a falhas mais inerentes ao questionário e entrevistas estruturadas, e especialmente aqueles compostos por questões fechadas para identificar a opinião dos respondentes, Bourdieu $(1982$, p.139) alertou que a falha principal desse tipo de levantamento seria "precisamente em colocar pessoas respondendo perguntas que elas não se perguntaram. " Por outro lado, Priestner e Borg (2016) contextualizam mais pragmaticamente a tendência pelo profissional bibliotecário optar por esses métodos: são relativamente baratos,

\begin{tabular}{l|l|} 
v.15 & n.3 \\
\hline
\end{tabular}

p.550-570

set./dez. 2017 
rápidos e de implementação simples. Além desses aspectos, dizem esses autores, o profissional bibliotecário usualmente não tem sido treinado no uso de outros métodos mais qualitativos, e possivelmente mais complexos e interpretativos.

Chowdhury e Chowdhury (2011) compara os métodos quantitativos e qualitativos nos estudos de usos e usuários, observando que os primeiros procuraram gerar dados sobre um fenômeno específico, capturando aquilo que acontece num dado momento, entendido a representar uma realidade objetiva e imparcial. Os métodos qualitativos, por outro lado, visam coletar informações do contexto em que os eventos ocorrem, procuram também descrever os eventos, e principalmente, da perspectiva dos atores participando naqueles eventos. Os métodos qualitativos procuram interpretar e descrever a complexidade e pluralismo no contex to do estudo, atribuindo centralidade do ponto de vista do usuário.

Como exemplo, tem-se a abordagem sense-making, apresentada em 1983 por Brenda Dervin, que encerra um conjunto de diretrizes conceituais e teóricas para análise da forma que as pessoas constroem sentido nos seus raciocínios e como fazem uso da informação e outros recursos nesse processo (DERVIN; NILAN, 1986). Ferreira (1997) desenvolve e revisa a ideia do sense-making como uma ferramenta útil e valiosa que os seres humanos procuram em suas tentativas de engrandecimento da própria vida, em sua busca de informação e conhecimento.

A observação é um instrumento de coleta de dados usada para a coleta de dados qualitativos na pesquisa dos estudos de usuários que envolve a observação e registro de eventos ou situações (CHOWDHURY; CHOWDHURY, 2011). De acordo com Richardson (1999), genericamente, a base de toda investigação no campo social é a observação, que pode ser utilizada em trabalhos científicos de quaisquer níveis, desde os estágios mais simples até os mais avançados, pois, "a observação, sob algum aspecto, é imprescindível em qualquer processo de pesquisa científica, pois ela tanto pode conjugar-se a outras técnicas de coleta de dados como pode ser empregada de forma independente e/ou exclusiva. " (RICHARDSON, 1999, p. 259). Em linguagem comum, observação é o exame minucioso sobre um fenômeno no seu todo ou em alguma de suas partes, captando precisamente o que se deseja do objeto examinado, entre outros significados da palavra. Em ciência, seu significado vai além disso, incorporando novos elementos ao sentido comum da palavra, apresentando uma dimensão mais ampla e complexa. Tradicionalmente, a observação é classificada como um método qualitativo de investigação e, como tal, sofre críticas positivas e negativas. Requer do pesquisador, entre outros cuidados, muita atenção, humildade, rigor metodológico e honestidade intelectual. Neste aspecto, Selltiz, Cook e Wrightsman (1987) referem-se à definição de observação:

[...] não é apenas uma das atividades mais difusas na vida diária: é também um instrumento básico da pesquisa científica. A observação torna-se uma técnica científica à medida que serve a um objetivo formulado de pesquisa, é sistematicamente planejada, sistematicamente registrada e ligada a proposições 
mais gerais e, em vez de ser apresentada como conjunto de curiosidades interessantes, é submetida a verificações e controles de validade e precisão. (SELLTIZ; COOK; WRIGHTSMAN, 1987, p. 45).

Para Nogueira (1969), o conhecimento começa com a observação e dela depende e todo indivíduo humano, na medida em que vive, também observa o mundo, tanto em seus aspectos físicos como sociais. Entretanto, uma observação espontânea, informal, pode apresentar uma série de desvantagens, do ponto de vista científico: 1) pode ser casual e esporádica; 2) o indivíduo observador pode perceber certos aspectos do mundo que o cerca e deixar de perceber outros; 3) o indivíduo pode não separar com nitidez o que resulta da observação, confundindo o que foi constatado do que foi inferido; 4) a atenção do indivíduo observador pode ser atraída mais pelo pitoresco do que pelo ordinário e comum que, frequentemente, passará despercebido; 5) neste tipo de observação, geralmente o indivíduo não faz anotações ou registros.

Como a observação espontânea é casual e esporádica, este ato de observar sem definição do objeto a ser observado pode se apresentar como uma forma positiva para o surgimento de novas ideias e objetos de estudo. Em vista disso, Nogueira (1969) considera como melhor solução o uso da observação sistemática, que pressupõe uma delimitação precisa no campo da investigação, tanto no tempo como no espaço. Nesse sentido, a observação sistemática permite ao indivíduo observador

[...] adquirir a capacidade de perceber o que é rotineiro, o que é usual, enfim, aquilo que a maioria deixa passar despercebido, quer por se ter tornado monótono e habitual, quer por se haver relegado para a esfera das observações que se costumam deixar subentendidas, implícitas, como assuntos de 'indiscutível' senso comum. (NOGUEIRA, 1969, p. 88).

Vieira (2013), Chowdhury e Chowdhury (2011) e Cunha (1982) questionaram o método de pesquisa observacional, destacando algumas desvantagens, tais como: a) o observador só pode observar certos comportamentos e aspectos físicos; b) não pode coletar informações sobre as motivações ou intenções; c) a amostra é tipicamente intencional, isto é, o pesquisador escolhe onde, como e a quem observar, para que não sejam generalizados os resultados para toda a população; d) o observador só pode coletar os dados quando estes ocorrem dentro de seu campo de observação; e) caso os usuários que estejam sendo observadas tenham conhecimento de que estão sob observação, podem alterar seu comportamento; f) falta de uniformidade, pois coisas diferentes podem ser observadas em momentos diferentes; g) o observador pode deixar de lado fatores importantes e ver o que não está preparado para ver; $h$ ) se envolver mais que um observardor (pesquisador), podem haver inconsistências nos registros entre eles.

Dentre as vantagens do método de observação, com base em Chowdhury e Chowdhury (2011) e Priestner e Borg (2016), podemos elencar:

- Provém experiência direta; 
- Por ser uma técnica relativamente simples, exige pouco treinamento (embora ajude se o observador esteja familiarizado com a organização ou atividade sendo observada);

- Provém discernimentos (insights) úteis no sistema existente;

- Não exige o investimento de tempo por parte do usuário.

Ao falar sobre os estudos de usuários da fase das décadas de 70, Wilson (2000) observou que os profissionais bibliotecários ficaram desapontados por estes estudos não terem gerado recomendações de mudanças na provisão de serviços e produtos. Esses estudos, predominantemente quantitativos, não retrataram os detalhes do contexto dentro do que os fenômenos de acesso e uso aconteceram, assim, não forneceram um quadro, imagem daquilo que realmente acontece com o usuário e por quê. No seu lugar, Wilson (2000) propõe:

\footnotetext{
A mudança de foco na pesquisa em ciência da informação para métodos qualitativos e pesquisa-ação daria subsídio à proposição de que a ciência da informação é uma ciência social, insistindo no uso mais inteligente dos métodos de pesquisa social para o desenvolvimento de modelos, do ponto de vista da filosofia da ciência social (e não física). (WILSON, 2000, p. ?).
}

Para Wilson (2000), os resultados de estudos de usuários que empregam métodos qualitativos, oriundos nas ciências sociais, podem ser candidatos a uma aplicação mais óbvia e imediata na organização a ser estudada. Tarapanoff, Araújo Junior e Cormier (2000) também chegaram a uma conclusão semelhante sobre a potencial "utilidade" dos resultados dos estudos de usuários em geral (tanto quantitativos quanto qualitativos), que, na concepção deles, devem ser considerados como uma ferramenta de gestão estratégica na biblioteca. Isto porque as necessidades informacionais do usuário poderão ser antecipadas e sua função excede a geração de dados quanto ao perfil do usuário e deve gerar indicadores que possam formular, principalmente, itens de controle de qualidade dos serviços prestados pela unidade de informação.

Os praticantes e proponentes da atual "virada etnográfica" dos estudos de usuários dentro da prisma da "UX: User Experience", embora reconheçam a complexidade e grande investimento de tempo e mão de obra necessários para os métodos etnográficos propostos, também claramente visualizam os potenciais usos tangíveis, pela organização, dos resultados de tais estudos. Como Laclos (2016) observa:

A pesquisa qualitativa é confusa (messy), na sua execução tal como nos dados que gera. A pesquisa qualitativa em bibliotecas tem dois propósitos principais: para melhorar as experiências dos usuários e para comunicar mais eficazmente com aqueles que financiam e apoiam as bibliotecas. (Laclos, 2016, p. 24-25).

Vale observar, também, que a parte dos estudos etnográficos com base em técnicas mais tradicionais, como a observação, podem acrescentar um contexto, por meio de uma camada de interpretação rica e densa, aos dados e estatísticas que um determinado setor da biblioteca já produza rotineiramente (JAMIESON, 2016). 


\section{PROCEDIMENTOS METOdOLógicos}

Apesar de ser classificada tradicionalmente como um método qualitativo, a observação também é quantificável e, para tal, não se deve apenas olhar e ver o fenômeno objeto de estudo, mas também estabelecer algumas condições prévias para seu desenvolvimento. (RICHARDSON, 1999). Em vista disto, os dados foram levantados em uma pesquisa de campo na forma de estudo de caso, em que o universo da pesquisa compreendeu o acervo de periódicos impressos da BCo, referentes ao seu uso no período de janeiro de 2012 a dezembro de 2015, incluindo a quantidade aplicada no atendimento de programas de comutação bibliográfica, tais como o COMUT.

A pesquisa consistiu-se nas seguintes etapas: 1) levantamento junto à BCo dos dados estatísticos de utilização dos periódicos impressos, no período compreendido entre os anos de 2012 a 2015; 2) elaboração de formulário para estruturação da pesquisa; 3) seleção de períodos de amostragem aleatoriamente; 4) observação dos usuários no setor de periódicos impressos, situado no piso 4 da $\mathrm{BCo}$; 5) coleta, análise e interpretação dos dados; 6) apresentação dos resultados.

Para a estruturação da pesquisa, foi elaborado um formulário para observação (Figura 1), de modo a não interferir no meio ambiente observado, caracterizando-a, portanto, como uma pesquisa sistemática (NOGUEIRA, 1969, p. 82). Foram observados os comportamentos que ocorreram espontaneamente em contextos naturais, sem participação no que aconteceu e sem nenhuma tentativa de manipular variáveis, pois a pesquisa foi do tipo não participante.

A coleta de dados para a pesquisa observacional ocorreu entre os dias 05 de abril e 10 de maio de 2016, totalizando 48 horas e 20 minutos de observação estruturada, sistemática e não participante, priorizando os horários de maior concentração de usuários, que é das 9 às 22 horas, segundo levantamento efetuado por Ninin et al (2015, p. 67) com base em 100.662 registros referentes ao empréstimo do acervo da BCo no ano de 2011. 


\begin{tabular}{|c|}
\hline PESQUISA OBSERVACIONAL - SETOR DE PERIÓDICOS IMPRESSOS - PISO 4, BCo/UFSCar \\
Data desta pesquisa/formulário: \\
\hline Horário: INÍCIO: _ TÉRMINO: \\
\hline ATIVIDADES e/ou ATITUDES observadas nos usuários:
\end{tabular}

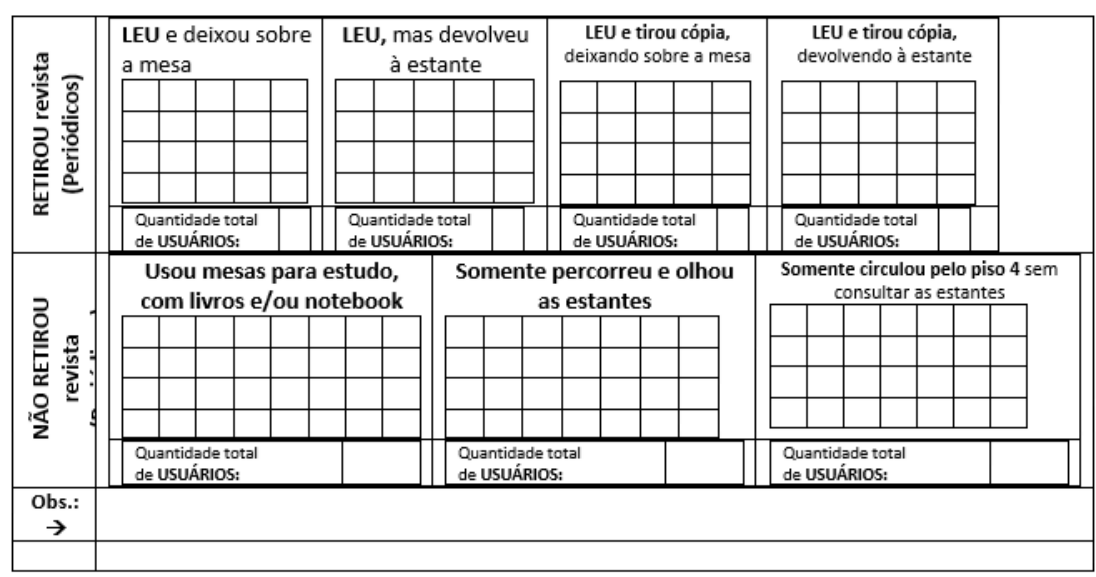

\begin{tabular}{|l|l|}
\hline Tempo total (horas) - no dia & \\
\hline Número de horas anterior: & \\
\hline Total de horas Acumulado: & \\
\hline
\end{tabular}

Figura 1 - Formulário elaborado e utilizado pelos autores para a estruturação da pesquisa observacional, em dias e horários alternados.

\section{DESCRIÇÃO E ANÁLISE DOS DADOS}

O levantamento dos dados compreendeu duas fases: a primeira focando as estatísticas de uso dos periódicos já acumuladas pela $\mathrm{BCo}$, com base na contagem do material deixado sobre as mesas. A segunda fase, realizada em 2016, foi a pesquisa observacional feita com usuários que frequentaram o setor de periódicos impressos, no piso 4 da BCo, identificando quantas fizeram uso dos periódicos e quantas somente utilizaram o espaço para seus estudos, sem consultar este acervo.

Os dados estatísticos apurados, na primeira fase da pesquisa, foram transcritos em planilhas de Microsoft Excel, cujo processamento obedeceu rigorosamente à descriminação e quantidades originais fornecidas pela BCo. O objetivo foi comparar valores de cada ano analisado, classificando os títulos pela frequência de uso em ordem decrescente. A partir desses resultados, foram elaborados gráficos para facilitar a leitura dos dados tabulados.

O setor de periódicos impressos da BCo conta atualmente com um acervo total de 4.187 títulos, de acordo com dados fornecidos pela própria Biblioteca. No período entre os anos de 2012 até 2015, analisado pela pesquisa, somente 798 títulos foram usados, incluindo o atendimento via COMUT, o que representa $19 \%$ do total. No Gráfico 1 demonstramos que, destes 798 títulos de periódicos usados, a maior parcela teve uma quantidade de acessos ou de uso mínima e os usos se dividiram, percentualmente, da seguinte forma: 


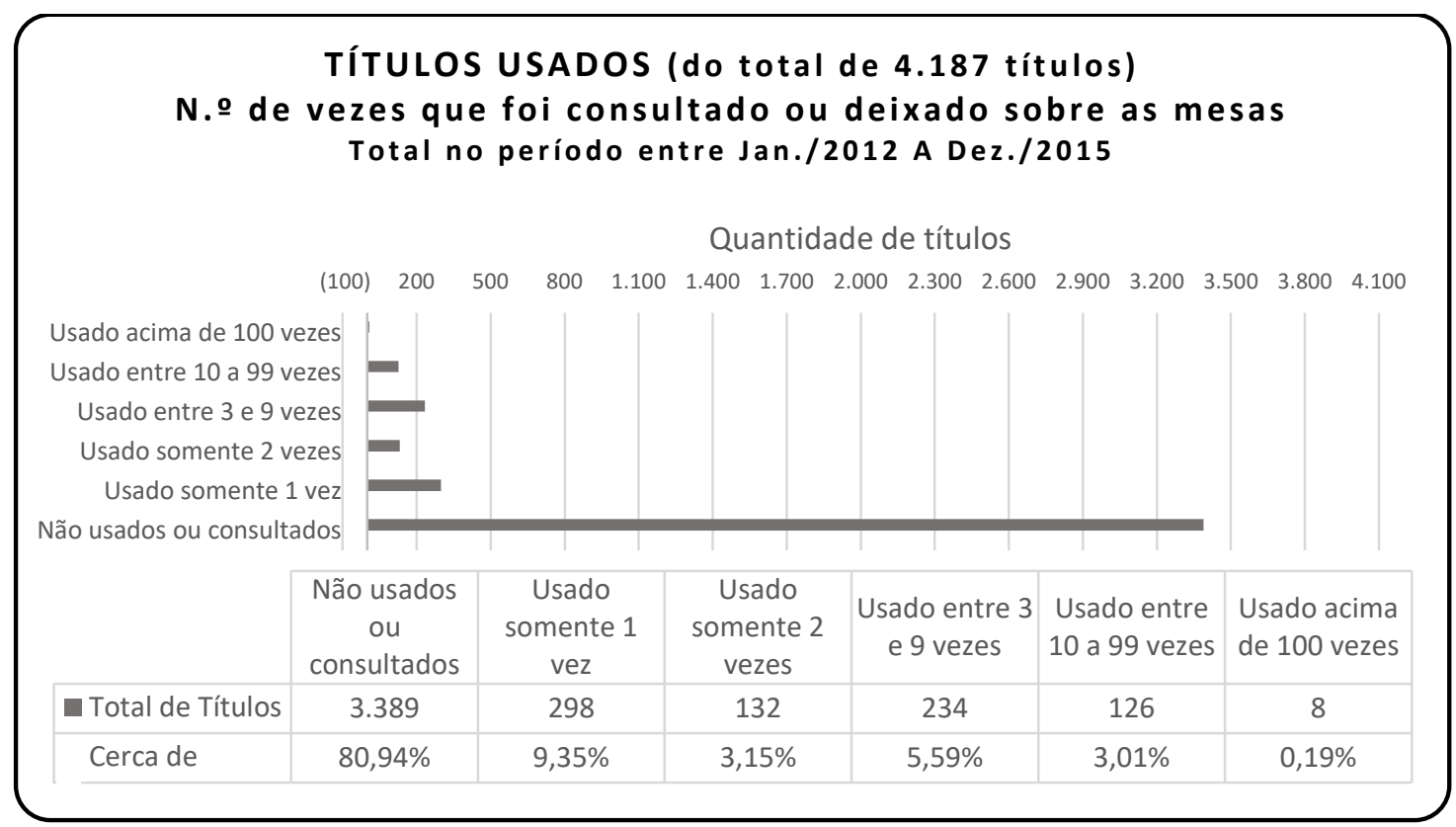

Gráfico 1 - Demonstração da quantidade de títulos usados, de acordo com o número de vezes que cada item foi consultado ou deixado sobre as mesas

Fonte: dados da pesquisa, 2016. Elaborado com base em estatísticas de contagem de mesa, referentes ao período de janeiro de 2012 a dezembro de 2015, disponibilizadas pela BCo (inclui atendimentos via COMUT).

Após 48 horas e 20 minutos de trabalho em campo foi identificada a quantidade de usuários que efetivamente retirou algum fascículo de periódico do setor sob análise. Esta quantidade mostrou-se muito aquém do esperado inicialmente. Também foi possível observar o comportamento dos frequentadores do setor de periódicos impressos no piso 4 da BCo.

O Gráfico 2 mostra o resultado de usuários que retiraram ou não fascículos de periódicos:

\section{SETOR DE PERIÓDICOS - PISO 4 - BCo. \\ Uso, consultas ou retiradas no setor (durante período total de observação: $48 \mathrm{~h}, 20 \mathrm{~min}$. )}

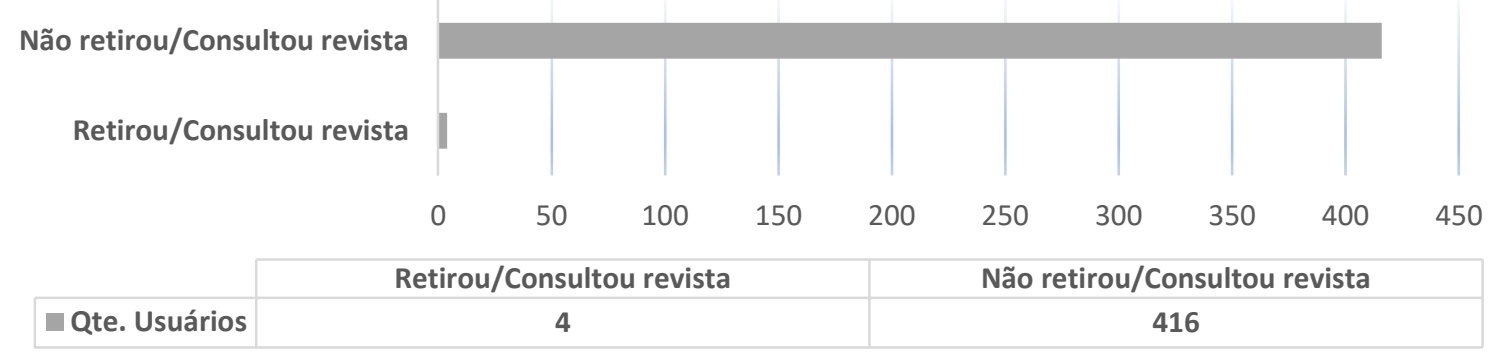

Gráfico 2 - Quantidade de usuários que retirou/usou periódicos

Fonte: dados da pesquisa, 2016. Elaborado com base na pesquisa observacional realizada entre os dias 04 de abril e 10 de maio de 2016, após 48 horas e 20 minutos de trabalho em campo. 


\section{RESULTADOS E DISCUSSÃo}

Analisando os resultados, observa-se que dos 4.187 títulos disponíveis no acervo, somente 798 títulos $(19,01 \%)$ foram usados durante o período estudado de 2012 a 2015, em contraposição a 3.389 títulos $(80,94 \%)$ não utilizados. Dentre estes 798 títulos usados no período, os 45 mais acessados são, principalmente, de revistas que não têm como objetivo editorial publicar artigos científicos, tais como: Veja; Época; Isto é; Superinteressante; Caros Amigos; Carta Capital; Isto é Dinheiro; Você S. A.; Kappa Magazine; Exame; Placar; entre outros títulos de revistas não acadêmicas. Além disso, dos 798 títulos utilizados no período, cerca de $69 \%$ também se refere a periódicos que não têm tradição na publicação de artigos científicos, tratando-se de revistas de interesse geral, segmentadas por público ou interesses profissionais específicos.

Confrontando o conjunto de dados estatísticos de uso com os levantados durante a pesquisa observacional, verificamos que é visível a queda gradual do uso de periódicos impressos, ano a ano, na BCo. Esta situação confirma que nesta Universidade também ocorrem os fatos constatados em outras instituições de ensino superior, conforme análise feita por COSTA (2007), em que os acessos ou consultas aos periódicos científicos estão cada vez mais sendo feitos por meio eletrônico pelo Portal de Periódicos CAPES, entre outros.

As estatísticas de acesso ao Portal de Periódicos CAPES, relativas à UFSCar, disponibilizadas pela CAPES (2016) no Sistema de Informações Georreferenciadas da CAPES (GEOCAPES), demonstram a linha de tendência na evolução dos acessos dos anos de 2011 a 2014, de acordo com demonstrado no Gráfico 3 (durante a elaboração do artigo, ainda não estavam disponíveis dados referentes a 2015).

Também verificamos que a maior parcela dos usuários (405 usuários de um total de 420) que frequentou o setor de periódicos impressos no piso 4 da $\mathrm{BCo}$, lá esteve com a intenção de usar o local para seus estudos e para uso da rede sem fio aberta da BCo, utilizando somente livros do acervo da biblioteca e/ou seus próprios notebooks. O material deixado sobre as mesas revelou que eram alunos de graduação ou pós-graduação das mais diversas áreas do conhecimento dos cursos oferecidos pela Universidade.

Houve preferência pelas mesas instaladas próximas às janelas, principalmente em dias de temperaturas mais elevadas. Nos dias com temperatura mais amena, os usuários dividiamse mais uniformemente pelas mesas disponibilizadas no recinto. A BCo tem uma quantidade de usuários muito expressiva sendo que, durante todo o expediente da biblioteca, há grande fluxo de usuários. Em certos momentos, os espaços disponibilizados para estudos parecem não ser suficientes para atender a demanda de usuários. Em vista disso, há desconforto e comentários entre os frequentadores da biblioteca ao ver o grande espaço ocupado no piso 4 , pelo acervo de periódicos impressos, que praticamente ninguém usa. 


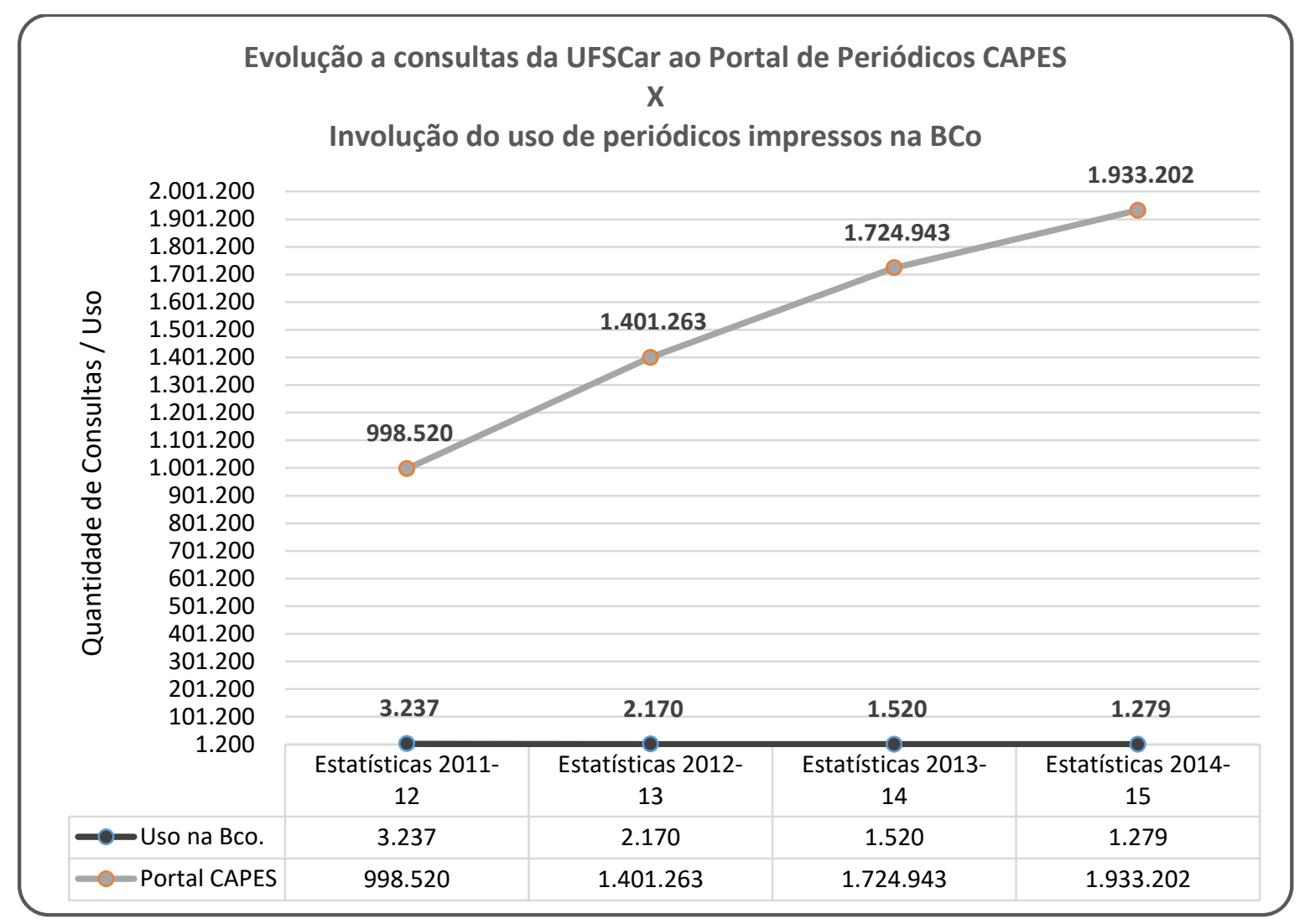

Gráfico 3 - Evolução anual da quantidade de acessos ao Portal de Periódicos CAPES - dados da UFSCar de 2011 a 2014

Fonte: Estatísticas Bco 2012-2016; GEOCAPES, 2016. Elaborado com base em dados disponíveis em GEOCAPES - Sistema de Informações Georreferenciadas |CAPES e estatísticas Bco.

Os resultados obtidos compreenderam um conjunto de indicadores que corroboraram a hipótese inicial de que, atualmente, os periódicos impressos da coleção BCo têm pouco uso. Isso não tem sido uma surpresa, já que o mesmo fenômeno vem ocorrendo em outras instituições de Ensino Superior em todo o mundo, segundo estudos de Liu (2005), Costa (2007), Breeding (2010), Oliveira (2013), Lown, Sierra e Boyer (2013), que estudaram as percepções dos usuários sobre recursos impressos e eletrônicos e as vantagens e desvantagens da evolução das revistas em seu formato eletrônico.

De posse desses indicadores, o gestor da Biblioteca talvez possa advogar e planejar com maior ênfase e embasamento, junto à Reitoria da Universidade, um espaço separado da BCo, para acolher e preservar o acervo de periódicos impressos, cedendo o nobre espaço ora ocupado no piso 4 da BCo para receber a ampliação do acervo de obras bibliográficas básicas das disciplinas oferecidas, cuja aquisição é feita regularmente em cumprimento à Lei n. ${ }^{\circ}$ 10.861 de 14 de abril de 2004 (BRASIL, 2004), que instituiu o Sistema Nacional de Avaliação da Educação Superior (SINAES), que é o instrumento de avaliação do ensino superior do Ministério da Educação (MEC). 
Além disso, um estudo comparativo entre os títulos de periódicos impressos que também se encontram on-line poderia identificar que boa parte do acervo impresso pode ser encontrada nas bases de dados, não havendo necessidade nem mesmo de acesso aos impressos, de modo que os mesmos podem ser armazenados apenas para fins de preservação.

É importante sempre observar os parâmetros de avaliação, verificando se a coleção satisfaz os usuários. Com o veloz desenvolvimento tecnológico, é preciso ficar atento às mudanças de interesse por parte da comunidade beneficiária da biblioteca, valendo-se de métodos de observação e outros estudos de usos e usuários, com os quais o profissional bibliotecário poderá inteirar-se das demandas dos usuários e seu grau de satisfação porque, mesmo após 85 anos, estas palavras ainda são verdadeiras: “[...] os livros são para usar. [...] a cada leitor seu livro. [...] a cada livro seu leitor. [...] poupe o tempo do leitor. [...] a biblioteca é um organismo em crescimento. [...]" (RANGANATHAN, 1931, p. 1-382, tradução nossa).

\section{CONCLUSÃO}

Este estudo buscou identificar, por meio de pesquisa observacional estruturada, sistemática e não participante, qual tendência de atuação os usuários demonstraram no setor de periódicos e quantos destes usuários fizeram uso/consulta de tal acervo. $\mathrm{O}$ estudo também se fundamentou em dados estatísticos de uso, disponibilizados pela BCo, baseados em contagem do material deixado sobre as mesas, pois, "[...] a maneira mais fácil de descobrir quais os itens ou tipos de itens que são consultados na biblioteca é examinar o material que foi deixado sobre as mesas, método este que é o adotado com mais frequência." (LANCASTER, 1993, p. 81).

Para a BCo e para a UFSCar, deixamos como sugestão o remanejamento dos periódicos impressos para outro local - mas não seu descarte - visto que seu uso vem decaindo de forma acentuada, ainda mais se considerarmos que, dos 4.187 títulos atualmente disponíveis no acervo, parte destes títulos também é disponibilizado por meio digital.

A direção da BCo, juntamente com seus servidores e colaboradores, vem envidando esforços para identificar quais títulos do acervo de periódicos impressos estão totalmente disponíveis no formato eletrônico, os quais a comunidade desta instituição de ensino superior pode acessar, por meio da rede UFSCar, sejam conteúdos pagos ou em acesso aberto. Deixamos em aberto a discussão sobre a temática da qualidade centrada no usuário de bibliotecas, bem como a importância de avaliar periodicamente como esta qualidade está sendo percebida pelos seus clientes.

Em recente publicação de Brito e Vergueiro (2013), estes autores destacam uma metodologia específica para este tipo de estudo, criada nos Estados Unidos e utilizada em centenas de bibliotecas ao redor do mundo, porém ainda pouco utilizado no Brasil: o método LibQUAL ${ }^{\circledR}$ que apresenta aspectos que permitem apoio à gestão de bibliotecas acadêmicas,

v.15

n.3

p.550-570
set./dez. 2017 
tais como a identificação de pontos fortes e fracos dos serviços prestados, sendo possível aproximar a biblioteca de seus clientes e realizar comparações com outras bibliotecas, além de ser uma ferramenta que pode contribuir para uma administração mais profissional das bibliotecas. A peça central do programa LibQUAL+ ${ }^{\circledR}$ é uma pesquisa baseada na Web com um conjunto de serviços oferecidos pela Association of Research Libraries (ARL) para a comunidade de bibliotecas, entre outros objetivos, de acordo com informações de site institucional do programa (LibQUAL+ $\left.{ }^{\oplus}, 2016\right)$.

A realização deste estudo, inicialmente, mostrou-se como um desafio complexo, porém, com a valiosa cooperação e subsídios da $\mathrm{BCo}$, a pesquisa se concluiu de maneira profícua, contribuindo na formação acadêmica em Biblioteconomia e Ciência da Informação. A oportunidade oferecida por este trabalho, sob a ótica do profissional bibliotecário, foi o enfoque diferenciado sobre o usuário, compatibilizando a literatura sobre usos e usuários com desenvolvimento de coleções e acervos, com administração de bibliotecas e unidades de informação e com levantamentos estatísticos de uso. $\mathrm{O}$ resultado aqui exposto, pela combinação destes conhecimentos, poderá contribuir, acima de tudo, na tomada de decisão administrativa de uma biblioteca ou unidade de informação.

\section{REFERÊNCIAS}

ALMEIDA, Maria Cristina Barbosa de. Planejamento de bibliotecas e serviços de informação. Brasília; Briquet de Lemos, 2000.

AOYAMA, Pâmela Tieme Barbosa. Estudo das necessidades de informação dos usuários da Biblioteca Digital do Senado Federal. Universidade de Brasília. Disponível em: < http://www2.senado.leg.br/bdsf/handle/id/84/browse?value=Aoyama\%2C+P\%C3\%A2mela+ Tieme+Barbosa\&type=author $>$. Acesso em: 02 abr. 2015.

BAPTISTA, Sofia Galvão; CUNHA, Murilo Bastos da. Estudo de usuários: visão global dos métodos de coleta de dados. Perspectivas em Ciência da Informação, Belo Horizonte, v. 12, n. 2, p. 168-184, maio/ago. 2007. Disponível em: < http://www.scielo.br/scielo.php?script=sci_arttext\&pid=S141399362007000200011\&lng=enee\&nrm=iso\&tlng=enee >. Acesso em: 20 abr. 2015.

BETTIOL, Eugênia Maranhão. Necessidades de informação: uma revisão. Revista de Biblioteconomia, Brasília, v. 18, n. 1, jan. /jun. 1990, p. 59-69. Disponível em: < http://basessibi.c3sl.ufpr.br/brapci/v/a/4649 >. Acesso em: 19 abr. 2015.

BOURDIEU, Pierre. A opinião pública não existe. In: THIOLLENT, Michel J.M. Crítica metodológica e investigação social e enquête operária. São Paulo: Polis, 1982. p. 137-153.

BRASIL. Presidência da República. Casa Civil. Subchefia para Assuntos Jurídicos. Lei n. ${ }^{\mathbf{0}}$ 10.861, de 14 de abril de 2004. Institui o Sistema Nacional de Avaliação da Educação Superior - SINAES. Disponível em: < http://www.planalto.gov.br/ccivil_03/_ato20042006/2004/lei/110.861.htm >. Acesso em: 21 maio 2016. 
BRASIL. Ministério da Educação. Fundação Capes. GEOCAPES Sistema de Informações Georreferenciadas | CAPES. Brasília, 2016. Disponível em: < http://geocapes.capes.gov.br/geocapes2/ >. Acesso em: 18 jun. 2016.

BREEDING, Marshall. The state of the art in library discovery. Computers in Libraries, v.30, n.1, 31-34. Disponível em: < http://www.librarytechnology.org/ltgdisplaytext.pl?RC=14574 >. Acesso em: 23 jun. 2016.

BRITO, Gisele Ferreira de; VERGUEIRO, Waldomiro de Castro Santos. Avaliação da qualidade da biblioteca acadêmica: a metodologia LibQUAL+ ${ }^{\circledR}$ e suas perspectivas de aplicação no Brasil. Brazilian Journal of Information Science: Research Trends, BJIS, Marilia (SP), v.7, n. Especial, p.26-46, 1º. Sem. 2013. Disponível em: < http://www2.marilia.unesp.br/revistas/index.php/bjis/article/download/3117/2403 >. Acesso em: 26 maio 2016.

CASE, Donald O. Looking for information: a survey of research on information seeking, needs and behavior. Library and Information Science Series. Edition: 3rd edition. Contributor(s): Donald O. Case (editor), Amanda Spink (series edited), 389 pp, Publication date: 19th April 2012. Imprint: Emerald Group Publishing Limited.

CHOO, Chun Wei. A organização do conhecimento: como as organizações usam a informação para criar significado, construir conhecimento e tomar decisões. São Paulo: Senac, 2000, 245 p.

CHOWDHURY, Gobinda G.; CHOWDHURY, Sudatta. Information users and usability in the digital age. London: Facet Publishing, 2011.

CONNOWAY, Lynn Silipigni, (Org.) The Library in the life of the user: engaging with people where they live and learn. Dublin, Ohio: OCLC Research, 2015. Disponível em: < http://www.oclc.org/content/dam/research/publications/2015/oclcresearch-library-in-life-of-

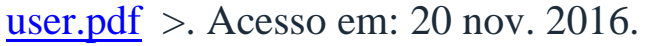

COSTA, Rubenildo Oliveira da. Análise do uso de periódicos científicos na transição do meio impresso ao eletrônico. 2007. 142p. Dissertação (Mestrado em Ciência da Informação) - Centro de Ciências Sociais Aplicadas, Pontifícia Universidade Católica de Campinas, Campinas. Disponível em: < http://www.bibliotecadigital.puccampinas.edu.br/tde_arquivos/2/TDE-2007-06-20T082105Z1336/Publico/Rubenildo\%20Oliveira\%20da\%20Costa-1.pdf >. Acesso em: 23 fev. 2016.

CUNHA, Murilo Bastos da. Metodologias para estudo dos usuários de informação científica e tecnológica. Revista de Biblioteconomia, Análise, Brasília, v. 1, n. 11, p. 1, jul./dez. 1982. Disponível em: < http://bogliolo.eci.ufmg.br/downloads/CUNHA_1982.pdf >. Acesso em: 27 fev. 2016.

DEMPSEY, Lorcan. From infrastructure to engagement: thinking about the library in the life of the user." Keynote presented at Minitex 24th ANNUAL INTERLIBRARY LOAN CONFERENCE, 12 May, 2015, in St. Paul. Disponível em: < http://www.slideshare.net/lisld/from-local-infrastructure-to-engagement-thinkingabout- 
the-library-in-the-life-of-the-user >. Acesso em: 20 nov. 2016.

DERVIN, Brenda; NILAN, Michael. Information needs and uses. Annual Review of Information Science and Technology ARIST, v. 21, 1986, p. 3-33. Disponível em: < https://comminfo.rutgers.edu/ tefko/Courses/612/Articles/zennezdervinnilan86arist.pdf $>$. Acesso em: 05 jun. 2016.

FERREIRA, Jose Rincon. A biblioteca digital. 1995. Disponível em:

<http://www.ime.usp.br/ is/infousp/rincon/rincon.htm>. Acesso em: 10 abr. 2015.

FIGUEIREDO, Nice Menezes de. Aspectos especiais de estudos de usuários. Ciência da Informação, Brasília, v. 12, n. 2, p. 43-57, jul. /dez. 1983. 112 p.

Disponível em: < http://revista.ibict.br/ciinf/index.php/ciinf/article/view/1496/1114 >. e também disponível em: < http:// http://revista.ibict.br/ciinf/article/view/184 >. Acesso em: 08 abr. 2015.

FIGUEIREDO, Nice Menezes de. Estudos de usos e usuários da informação. Brasília: IBICT, 1994. 154p. http://livroaberto.ibict.br/handle/1/452 . Disponível em:

$<$ https://www.google.com.br/url?sa=t\&rct=j\&q=\&esrc=s\&source=web\&cd=1\&cad=rja\&uact=8\&ved $=0$ ahUKEwjlxN7Um9XMAhXHEZAKHaJ_BSEQFggcMAA\&url=http\%3A\%2F\%2Flivroaberto.ibic t.br\%2Fbitstream\%2F1\%2F452\%2F1\%2FEstudos\%2520de\%2520uso\%2520e\%2520usu\%25C3\%25 A1rio.pdf\&usg=AFQjCNEXaJliSkSEWUkY3wm71ovtRC_f_g\&bvm=bv.121658157,d.Y2I>. Acesso em: 30 abr. 2016.

GONÇALVES, Ana Lúcia Ferreira. Gestão da informação na perspectiva do usuário: subsídios para uma política em bibliotecas universitárias. Rio de Janeiro: Interciência, 2013, $156 \mathrm{p}$.

JAMIESON, Helen. Spaces for learning?: using ethnographic techniques: a case study from the University Library, Edge Hill University. In: PRIESTNER, Andy; BORG, Matt. (Orgs). User experience in libraries. Applying ethnography and human-centred design. New York/London: Routledge, 2016. pp.173-177.

JANES, Mark. Time to take another Bath? a preliminary report on the feasibility of repeating the INFROSS study. Disponível em: < https://www.ifla.org/time-to-take-anotherbath >. Acesso em: 08 abr. 2015.

LACLOS, Donna M. Embracing an ethnographic agenda. Context, collaboration, and complexity. In: PRIESTNER, Andy; BORG, Matt. (Orgs). User experience in libraries. Applying ethnography and human-centred design. New York/London: Routledge, 2016. pp.21-37.

LANCASTER, Frederick Wilfrid. Avaliação de serviços de biblioteca. Brasília: Briquet de Lemos, c1993. 356p.

LIBQUAL $+{ }^{\circledR}$, Charting Library Service Quality. General information: what is LibQUAL $+{ }^{\circledR}$ ? 2016. Disponível em: < https://www.libqual.org/about/about_lq/general_info >. Acesso em: 26 maio 2016. 
LIMA, Maria Lecticia de Andrade. Usuários de uma biblioteca universitária: estudo realizado no Instituto de Filosofia e Ciências Humanas da Universidade Federal de Pernambuco.

Ciência da Informação, Rio de Janeiro: v. 3 n. 1, 1974, p. 51-56. Disponível em: < http://revista.ibict.br/index.php/ciinf/article/view/1688/1293 >. Acesso em: 11 abr. 2015.

LIU, Ziming. Print vs. electronic resources: a study of user perceptions, preferences, and use. Information Processing \& Management, v.42, n.2, pp.583-592, 2006.

LOWN, Cory, SIERRA, Tito, BOYER, Josh. (2013). How users search the library from a single search box. College \& Research Libraries, v.74, n.3, p.227-241. Disponível em: < http://crl.acrl.org/content/74/3/227.full.pdf >. Acesso em 22 jun. 2015.

MIRANDA, Silvânia. Como as necessidades de informação podem se relacionar com as competências informacionais. Ciência da Informação, Brasília, v. 35, n. 3, p. 99-114, set. /dez. 2006. Disponível em: < http://www.scielo.br/pdf/ci/v35n3/v35n3a10.pdf >. Acesso em: 02 abr. 2015.

NININ, Débora Marroco; AMARAL, Roniberto Morato do; MILANEZ, Douglas Henrique; FARIA, Leandro Innocentini Lopes de. Indicadores de circulação do acervo na Biblioteca Comunitária da Universidade Federal de São Carlos. Transinformação [online]. 2015, v. 27, n. 1, p. 59-71. ISSN 0103-3786. Disponível em: < http://www.brapci.ufpr.br/brapci/index.php/article/view/0000016245/d79f5573017dbba000ed $\underline{4 \mathrm{~d} 123 \mathrm{~d} 8234 \mathrm{e} 7}>$. Acesso em: 02 mar. 2016.

NOGUEIRA, Oracy. Pesquisa social: introdução às suas técnicas. 2. ed. São Paulo: Nacional, 1969. 209 p. (Biblioteca Universitária série Ciências Sociais v.26).

OLIVEIRA, Caroline Brito de. Periódicos impressos X eletrônicos: convivência de suportes de informação no Banco Nacional de Desenvolvimento Econômico e Social - BNDES.

Revista digital do BNDES. 2013. Disponível em: <https://web.bndes.gov.br/bib/jspui/handle/1408/2201>. Acesso em: 23 fev. 2016.

PADILHA, Roberto de Queiroz (org.); LIMA, Valéria Vernaschi (org.). Curso de Medicina. Ciclo: Integralidade do Cuidado I - $1^{\circ}$ Ano. Caderno do Curso de Medicina. Site Institucional da Universidade Federal de São Carlos. Disponível em: < http://www2.ufscar.br/vidaacademica/cmedicina/curso_medicina.pdf $>$. Acesso em: 05 abr. 2015.

PITHAN, Denise Nunes. Diversidade cultural em usabilidade de bibliotecas digitais: análise de literatura. 2009. 150 f. Dissertação (mestrado) - Universidade de São Paulo, São Paulo. Disponível em: < http://www.teses.usp.br/teses/disponiveis/27/27151/tde-25102010164838/en.php >. Acesso em: 10 abr. 2015.

PRIESTNER, Andy; BORG, Matt. Uncovering complexity and detail. The UX proposition. In: PRIESTNER, Andy; BORG, Matt. (Orgs). User experience in libraries. Applying ethnography and human-centred design. New York/London: Routledge, 2016, p. 1-8. 
RANGANATHAN, Shiyali Ramamrita. The five laws of library science, by S. R.

Ranganathan, with a foreword by Sir P. S. Sivaswami Aiyer and an introduction by W. C. Berwick Sayers. Madras, The Madras Library Association; 1931. Disponível em: < http://courseweb.lis.illinois.edu/ katewill/fall2014-

502/ranganathan\%201931\%20five\%20laws\%20lib\%20science\%20uc1.\$b99721.pdf $>$.

Acesso em: 06 mar. 2016.

RIBEIRO, Luciane Meire; COSTA Luzia Sigoli. Estudos de uso e usuários da informação: uma análise do foco e dos tipos de grupos estudados historicamente e suas relações com as tendências atuais. In: CONGRESSO BRASILEIRO DE BIBLIOTECONOMIA E CIÊNCIA DA INFORMAÇÃO, 24, 2011, Porto Alegre. Anais... Porto Alegre: FEBAB, 2011.

Disponível em: < www.febab.org.br/congressos/index.php/cbbd/xxiv/paper/.../428 >. Acesso em: 31 mar. 2015.

RICHARDSON, Roberto Jarry. Pesquisa social: métodos e técnicas. São Paulo: Atlas, 1999. $344 \mathrm{p}$.

SELLTIZ, Claire; COOK, Stuart Wellford; WRIGHTSMAN, Lawrence Samuel. Métodos de pesquisa nas relações sociais. 2. ed. São Paulo: EPU, 1987. 67 p.

TARAPANOFF, Kira; ARAÚJO JÚNIOR, Rogério Henrique de; CORMIER, Patrícia Marie Jeanne. Sociedade da informação e inteligência em unidades de informação. Ciência da Informação, Brasília, v. 29, n. 3, p. 91-100, set. /dez. 2000. Disponível em: <http://revista.ibict.br/index.php/ciinf/article/view/231/206>. Acesso em: 02 abr. 2015.

UNIVERSIDADE FEDERAL DE SÃO CARLOS. Biblioteca Comunitária. Apresentação. Disponível em: <

http://www2.ufscar.br/interface_frames/index.php?link=http://www.bco.ufscar.br $>$. Acesso em: 19 mar. 2016.

VIEIRA, Sonia. Pesquisas observacionais. 2013. Disponível em: < http://soniavieira.blogspot.com.br/2013/12/pesquisas-observacionais 5865.html >. Acesso em: 13 abr. 2016.

WILSON, Thomas D. Recent trends in user studies: action research and qualitative methods. Information Research, v.5, n.3, 2000. Disponível em: < http://www.informationr.net/ir/53/paper76.html > . Acesso em: 3 abr. 2015.
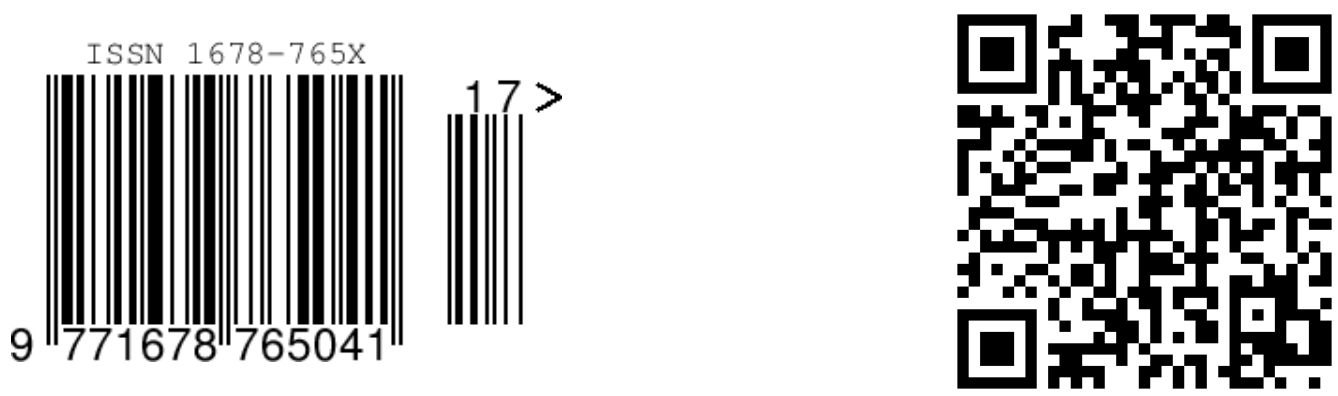